\title{
س
}

$>\mathrm{DE}$

름AGOGIE

Recherches en éducation

164 | juillet-septembre 2008

Évaluation des politiques éducatives et comparaisons internationales

\section{GOMBERT Philippe. L'École et ses stratèges, Les pratiques éducatives des nouvelles classes supérieures}

Rennes : Presses universitaires de Rennes, 2008. - 250 p.

Tristan Poullaouec

\section{OpenEdition}

Édition électronique

URL : http://journals.openedition.org/rfp/2068

DOI : $10.4000 /$ rfp. 2068

ISSN : 2105-2913

Éditeur

ENS Éditions

Édition imprimée

Date de publication : 1 juillet 2008

Pagination : 167-169

ISBN : 978-2-7342-1136-5

ISSN : 0556-7807

Référence électronique

Tristan Poullaouec, « Gombert Philippe. L'École et ses stratèges, Les pratiques éducatives des nouvelles classes supérieures », Revue française de pédagogie [En ligne], 164 | juillet-septembre 2008, mis en ligne le 14 octobre 2010, consulté le 22 septembre 2020. URL : http://journals.openedition.org/rfp/2068 ;

DOI : https://doi.org/10.4000/rfp.2068

Ce document a été généré automatiquement le 22 septembre 2020.

(c) tous droits réservés 


\title{
GOMBERT Philippe. L'École et ses
}

\section{stratèges, Les pratiques éducatives des nouvelles classes supérieures}

Rennes : Presses universitaires de Rennes, 2008. - 250 p.

\author{
Tristan Poullaouec
}

\section{RÉFÉRENCE}

GOMBERT Philippe. L'École et ses stratèges, Les pratiques éducatives des nouvelles classes

supérieures. Rennes : Presses universitaires de Rennes, 2008. - 250 p.

1 Issu d'une thèse de doctorat en sociologie (Gombert, 2006), ce livre se propose « d'explorer les stratégies scolaires des fractions inférieures des classes supérieures » et « de montrer quelles sont [leurs] conséquences en termes de régulation des pratiques éducatives" (p.10). Pour ce faire, il s'appuie d'une part sur une soixantaine d'entretiens menés auprès de cadres supérieurs du privé à Rueil-Malmaison, dans la banlieue ouest de Paris, et d'autre part sur une histoire nationale et locale des associations de parents d'élèves réalisée à partir d'une synthèse des travaux existants, d'entretiens avec des responsables associatifs et de documents publiés par les trois grandes fédérations ${ }^{1}$. Ces matériaux ont été pour partie élaborés dans le cadre d'une étude sur la ségrégation urbaine et scolaire dans le département des Hauts-de-Seine (Oberti \& van Zanten, 2004). Le travail de P. Gombert présente ainsi l'intérêt de tourner le regard scientifique vers des catégories sociales relativement peu étudiées par la sociologie de l'éducation, alors qu'elles investissent de plus en plus la scène scolaire en lieu et place des cadres du public, notamment à travers des associations locales de parents d'élèves qui se définissent volontiers comme autonomes et apolitiques pour mieux se distancier des grandes fédérations aux idéologies plus marquées.

2 La première partie de l'ouvrage se présente comme " une approche socio-historique des associations de parents d'élèves ». S'inspirant d'une typologie développée par 
B. Charlot pour caractériser les rapports entre les pouvoirs publics et l'école, elle décompose l'analyse en trois chapitres historiques. Jusqu'aux années soixante-dix, les associations de parents d'élèves prennent leur essor en réaction aux tensions suscitées par le projet laïque de l'État « éducateur ", qui tient les parents à distance de l'école : la PEEP dès 1910, l'UNAPEL en 1933 et la FCPE en 1947 (chapitre I). L'entrée dans la crise économique au milieu des années soixante-dix ouvre une seconde période au cours de laquelle l'État accepte de devenir "animateur» d'un partenariat entre les parents et l'école. La montée parallèle des nouvelles couches moyennes salariées des secteurs publics et du libéralisme culturel coïncident avec l'intégration des thèmes de l'autonomie individuelle, de l'épanouissement des élèves et de la participation des parents dans les affaires scolaires (chapitre II). Selon P. Gombert, c'est enfin un État "pragmatique» qui doit faire face à partir des années quatre-vingt aux attentes croissantes des parents et à la demande d'efficacité qu'ils adressent de plus en plus à l'école à travers les associations de parents d'élèves, dans une logique de consommateurs. Au final, si la FCPE reste largement majoritaire aux élections de parents d'élèves dans les écoles, sa domination s'effrite au profit des associations locales (chapitre III).

3 La seconde partie est consacrée aux "stratégies éducatives des nouvelles classes supérieures». Si l'auteur semble hésiter dans sa caractérisation sociologique de la population étudiée, évoquant tantôt les classes moyennes, tantôt la nouvelle bourgeoisie, parfois de façon plus précise les managers mais souvent aussi de façon moins définie les travailleurs de "la nouvelle économie», le lecteur identifie facilement dans les extraits d'entretiens les parents appartenant au groupe des cadres supérieurs des secteurs privés ou membres des professions libérales. La description morphologique de son terrain au chapitre IV permet à $\mathrm{P}$. Gombert de mieux spécifier ces positions sociales en les inscrivant dans les quartiers huppés des sièges sociaux de grandes entreprises. Elle souligne également les rapports étroits entre l'offre scolaire locale, les politiques urbaines et la "gentrification». Les chapitres suivants sont sans doute les plus intéressants pour l'illustration qu'ils fournissent des deux préoccupations centrales de l'éducation familiale dans ces "nouvelles classes supérieures » : l'idéal d'autonomie et d'épanouissement individuel d'un côté (chapitre v), le culte de la performance et les stratégies scolaires de long terme de l'autre (chapitre VI). On peut toutefois regretter que les tensions entre ces principes de socialisation ne soient pas mieux examinées ici, probablement faute d'avoir rapporté les discours des enquêtés aux trajectoires et expériences scolaires de leurs enfants.

Mais il est vrai que ce n'est pas l'objet principal du livre, qui est finalement traité dans la troisième partie portant sur les interventions locales des parents d'élèves dans la gestion des affaires scolaires (chapitre viI) et sur l'émergence d'une logique pragmatique dans la régulation des politiques éducatives (chapitre vIII). Décrits comme de véritables "professionnels ", très avertis des savoirs d'expertise et n'hésitant pas à intervenir dans les écoles et les collèges pour faire valoir leur prétention à devenir de "véritables acteurs des politiques éducatives", les jeunes cadres de la nouvelle économie se regroupent de plus en plus à Rueil-Malmaison dans une association locale de parents indépendants, dépolitisés et revendiquant tout à la fois la convivialité et l'efficacité dans leur "management» des affaires scolaires. Leur valorisation du pragmatisme trouve l'occasion de s'exprimer pleinement dans la nouvelle logique partenariale des politiques éducatives municipales, notamment autour des thèmes 
consensuels de la sécurité et de la délinquance. Les associations de parents défendent alors «les activités de médiation» entre les diverses institutions locales dont elles critiquent par ailleurs volontiers le bureaucratisme.

5 L'ouvrage de P. Gombert intéressera sans aucun doute beaucoup les lecteurs souhaitant mieux comprendre les transformations en cours dans les politiques scolaires, au plan local comme au plan national, voire international, comme le suggère habilement l'auteur en se référant en conclusion aux travaux de C. Maroy (2006). Il laisse en revanche sur leur faim ceux qui en attendent aussi une meilleure compréhension des stratégies de transmission des savoirs scolaires pourtant intensément à l'œuvre dans ces nouvelles familles bourgeoises. Puisque ces dernières n'hésitent pas à remettre en cause la légitimité pédagogique des enseignants, il aurait été instructif d'étudier concrètement les pratiques d'enseignement que développent ces parents d'élèves hors de l'école. Second bémol, le statut des matériaux d'enquête exposés est parfois indécis, comme lorsqu'un bulletin de la PEEP dénonçant «l'absence de liberté d'opinion » au sein de la FCPE vient apparemment appuyer une analyse de P.Gombert sur le fonctionnement idéologique et politique de ladite FCPE (p. 43). Enfin, s'il est dommage que la question de la généralisation des résultats ne soit jamais véritablement posée par l'auteur, le lecteur y trouvera cependant des conclusions souvent tout à fait convergentes avec celles des travaux de M. Barthélemy sur les associations de parents d'élèves (Barthélémy, 1995) ou de M. Pinçon et M. Pinçon-Charlot sur les écoles primaires des beaux quartiers (Pinçon \& Pinçon-Charlot, 1989).

\section{BIBLIOGRAPHIE}

BARTHÉLÉMY M. (1995). « Des militants de l'école. Les associations de parents d'élèves en France ». Revue française de sociologie, vol. $36, \mathrm{n}^{\circ} 3, \mathrm{p} .439-472$.

GOMBERT P. (2006). Pragmatisme, éducation et classes moyennes. Le cas des associations de parents d'élèves. Thèse de doctorat en sociologie, Institut d'études politiques de Paris.

OBERTI M. \& VAN ZANTEN A. (2004). Ségrégation urbaine et scolaire : le cas du département des Hauts-deSeine. Paris : Observatoire sociologique du changement.

MAROY C. (2006). École, régulation et marché. Une comparaison de six espaces scolaires locaux en Europe. Paris : PUF.

PINÇON M. \& PINÇON-CHARLOT M. (1989). Dans les beaux quartiers. Paris : Seuil.

\section{NOTES}

1. La fédération des parents d'élèves de l'enseignement public, l'union nationale des parents d'élèves de l'école libre et la fédération des conseils de parents d'élèves. 


\section{AUTEURS}

TRISTAN POULLAOUEC

Centre nantais de Sociologie

Université de Nantes 